\title{
Collagenous sprue is not always associated with dismal outcomes: a clinicopathological study of 19 patients
}

\author{
Efsevia Vakiani ${ }^{1}$, Carolina Arguelles-Grande ${ }^{2}$, Mahesh M Mansukhani ${ }^{3}$, Suzanne K Lewis ${ }^{2}$, \\ Heidrun Rotterdam ${ }^{3}$, Peter $\mathrm{H}_{\text {Green }}{ }^{2}$ and Govind Bhagat ${ }^{3}$
}

\begin{abstract}
${ }^{1}$ Department of Pathology, Memorial Sloan-Kettering Cancer Center, New York, NY, USA; ${ }^{2}$ Department of Medicine, Columbia University, New York, NY, USA and ${ }^{3}$ Department of Pathology, Columbia University,
\end{abstract} New York, NY, USA

\begin{abstract}
Collagenous sprue is associated with high morbidity; however, the etiology of this disorder is unclear. Data regarding the pathological and clinical manifestations of patients with collagenous sprue are also limited. We, thus, undertook this study to gain insight into the etiology, disease manifestations and outcomes of collagenous sprue. We searched our departmental database (1999-2008) to identify cases of collagenous sprue and to obtain clinical and laboratory data. Small bowel histology, including thickness of subepithelial collagen, intra-epithelial lymphocyte phenotype and results of T-cell clonality assays were evaluated. Nineteen patients (15 women, 4 men, age 22-80 years, mean 57 years) were identified. Seventeen (89\%) had celiac disease and two had unclassified sprue; 9 of $17(53 \%)$ celiac disease patients had refractory disease; 5 of $15(33 \%)$ lacked diarrhea (atypical presentation), including 2 of $6(33 \%)$ with active (untreated) celiac disease and 3 of $9(33 \%)$ with refractory celiac disease. Autoimmune disorders were seen in 12 of $19(63 \%)$ patients and microscopic colitis $(n=7)$, lymphocytic gastritis $(n=2)$ or collagenous gastritis $(n=2)$ were seen in nine patients. Subepithelial collagen thickness was mildly $(n=6)$, moderately $(n=10)$, or markedly $(n=3)$ increased and villous atrophy was total $(n=13)$ or subtotal $(n=6)$. Phenotypically aberrant intraepithelial lymphocytes were not detected in any case. Polymerase chain reaction analysis showed a dominant T-cell clone in the only patient with refractory celiac disease type II. Histological improvement occurred in 7 of $11(64 \%)$ patients. Overall, 8 of $19(42 \%)$ responded to gluten-free diet, including 2 of $9(22 \%)$ with refractory celiac disease and 10 responded to immunomodulatory therapy, including 6 of $9(67 \%)$ with refractory celiac disease. Only one patient died from complications of refractory celiac disease. No patient developed lymphoma. The vast majority of our patients with collagenous sprue had celiac disease. Although, many patients required immunomodulatory therapy for symptom control, a subset responded to gluten-free diet alone. In our experience, collagenous sprue patients had relatively good clinical outcomes.
\end{abstract}

Modern Pathology (2010) 23, 12-26; doi:10.1038/modpathol.2009.151; published online 23 October 2009

Keywords: collagenous sprue; celiac disease; subepithelial fibrosis; refractory sprue; intraepithelial lymphocytes; phenotype

Collagenous sprue is a clinicopathological entity characterized by diarrhea and malabsorption accompanied by the histological findings of subepithelial collagen deposition and severe villous atrophy of

Correspondence: Dr G Bhagat, MD, Department of Pathology, Columbia University Medical Center, VC14-228, 630W 168th Street, New York, NY 10032, USA.

E-mail: gb96@columbia.edu

Received 15 May 2009; revised 6 August 2009; accepted 11 August 2009; published online 23 October 2009 small bowel mucosa. The term collagenous sprue was first introduced by Weinstein et $a l^{2}$ in 1970, although descriptions of subepithelial deposits of eosinophilic hyaline material, consistent with collagen, date back to 1947., ${ }^{1,2}$ Only isolated case reports and small series have been published since then. ${ }^{3-16}$

The occurrence of collagenous sprue has been reported in individuals with celiac disease, tropical sprue, milk intolerance, myotonic dystrophy, malignancy-related paraneoplastic syndrome, and common variable immunodeficiency. ${ }^{4,5,10,11,14,17,18}$ These 
studies have suggested that subepithelial collagen deposition might be a non-specific feature.

Regardless of the etiology, collagenous sprue is thought to portend a poor prognosis with severe morbidity and mortality ${ }^{2,4,6,11,13}$ and has been described as a manifestation of refractory sprue. ${ }^{5,11} \mathrm{~A}$ few studies, however, have reported a good response to treatment and favorable outcomes. ${ }^{4,8-10}$ The discrepant findings have been attributed to inclusion of cases with mild alterations of the subepithelial collagen table in some studies, but currently there are no well-defined histological criteria for diagnosing collagenous sprue.

The aim of our study was to describe the etiology of collagenous sprue and provide objective histological criteria for diagnosis. We also assessed the relationship between the extent of subepithelial collagen deposition in small bowel biopsies and other pathologic findings, clinical presentation, and disease outcomes of individuals with collagenous sprue seen at our institution.

\section{Materials and methods}

\section{Case Selection}

We searched our departmental database to identify patients with increased subepithelial collagen documented on a review of their small bowel biopsies by HR, GB, and PHG, over a 10-year period (January 1999-December 2008). In the absence of established criteria regarding the thickness of small bowel subepithelial collagen required for diagnosing collagenous sprue, small bowel biopsies from two groups of control patients (individuals lacking small bowel pathology and untreated celiac disease patients), evaluated at our center during the study interval, were also assessed in order to establish the threshold (or 'cutoff' value) for 'normal' and celiac disease-related changes in subepithelial collagen thickness.

\section{Clinical Data}

Clinical data, including results of serology, HLA-DQ type, presence of other immune or autoimmune conditions, symptoms at presentation, endoscopic findings, type of treatment, response to treatment, and outcome were collected from the treating physicians. Symptoms were classified as classical (diarrhea predominant) or atypical (anemia, bone loss or screening). ${ }^{19}$ The clinical presentations of celiac disease were classified as (1) active celiac disease (patients with untreated celiac disease), (2) patients on gluten-free diet, and (3) refractory celiac disease (disease refractory to gluten-free diet for at least 6 months). The latter was further subclassified as primary or secondary, based on the absence or presence of previous response to gluten-free diet, and as type I or type II, based on the presence of a polyclonal or clonal population of intraepithelial lymphocytes. ${ }^{20-22}$ Cases where the underlying etiology could not be ascertained were designated as unclassified sprue. This study was approved by our Institutional Review Board.

\section{Histologic Evaluation}

Hematoxylin- and eosin-stained sections of all diagnostic and follow-up small bowel biopsies, and gastric and colonic biopsies, were evaluated where available. The degree of villous atrophy was classified as partial, subtotal and total corresponding to Marsh stages 3a, 3b and 3c, respectively, as described previously. ${ }^{23}$ Presence of a thickened subepithelial collagen layer, intraepithelial lymphocytosis, epithelial damage (eg, cytoplasmic vacuolization, loss of columnar shape), detachment of surface epithelium, and severity of lamina propria chronic inflammation (mild-inflammatory cells occupying $1 / 3$ of the lamina propria; moderateinflammatory cells occupying $2 / 3$ of the lamina propria; marked-entire lamina propria occupied and distended by inflammatory cells) were recorded. Presence and distribution of neutrophils was noted. The number of eosinophils was counted in 10 random high-power fields and the mean per high power field was calculated.

Masson trichrome-stained sections were used to evaluate and measure the subepithelial collagen thickness in all small bowel biopsies using an ocular micrometer (magnification $\times 600$ ) and an Olympus BX41 microscope (Olympus America, Center Valley, PA, USA). Three different fields were measured in well-oriented biopsies and mean thickness was calculated, tendrils of collagen extending into the lamina propria were not included in the measurement. Distribution of subepithelial collagen was classified as patchy if only a subset of biopsy pieces showed increased collagen or if only portions of the biopsy pieces showed increased collagen and diffuse if all biopsies showed increased subepithelial collagen along their entire length.

\section{Phenotypic Analysis}

Immunohistochemical stains were performed with antibodies against CD3, CD8 and smooth muscle antigen (DAKO, Carpinteria, CA, USA) on paraffin sections after heat-induced antigen retrieval using an autostainer (Universal staining system, DAKO) according to standard methods. The Envision Plus kit (DAKO) was used for detection. CD3 + and CD8 + intraepithelial lymphocytes were counted per 500 enterocytes. Intraepithelial lymphocytosis was graded as mild (30-50 intraepithelial lymphocytes/100 enterocytes), moderate (51-75 intraepithelial lymphocytes/100 enterocytes) or marked ( $>75$ intraepithelial lymphocytes/100 enterocytes). Cases were deemed to have a significant expansion of 
CD8- intraepithelial lymphocytes, if the percentage of CD3 + lymphocytes expressing CD8 was $\leqslant 50 \%$.

Four-color flow cytometric analysis (FACScan; BD Biosciences, San Diego, CA, USA) was performed on

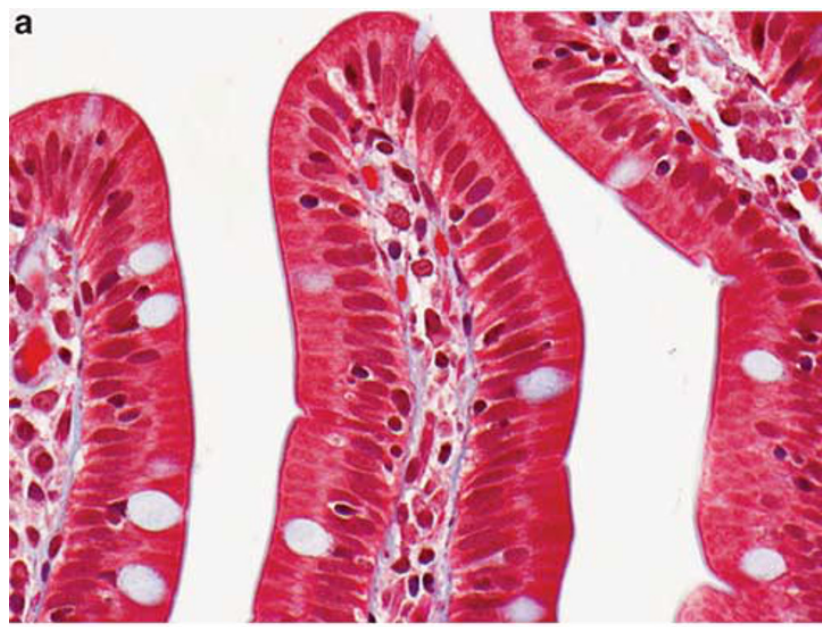

b

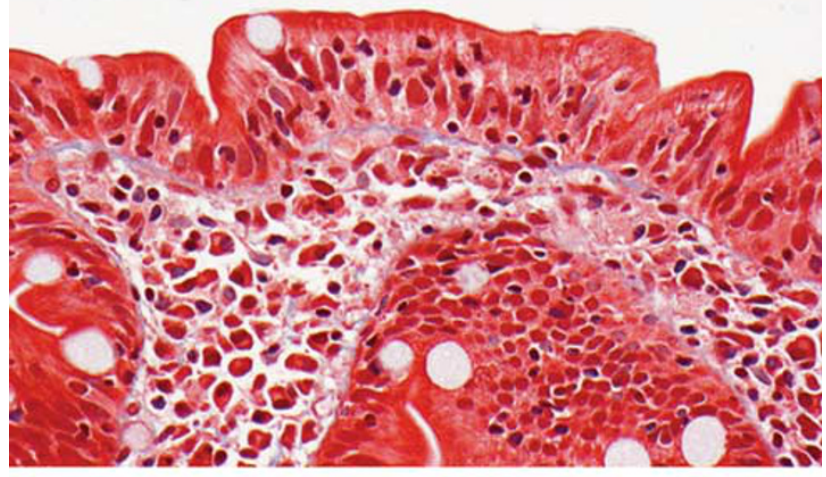

c

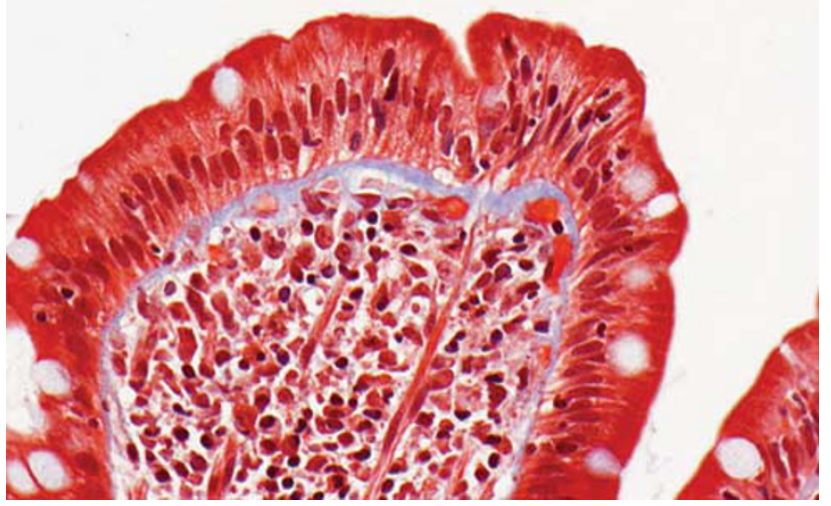

Figure 1 Trichrome-stained sections show thin BM in normal controls $(\mathbf{a}, \times 400)$ and some active celiac disease patients (b, $\times 400$ ). Minimal thickening of the BM was seen in $60 \%$ of the biopsies from active celiac disease patients $(\mathbf{c}, \times 400)$. small bowel biopsies using a panel of antibodies against $\mathrm{T}$ - and NK-cell antigens, and CD103 as described. $^{24}$ Data were analyzed using the Cell Quest software (BD Biosciences).

\section{T-Cell Receptor Gene Rearrangement Analysis}

Polymerase chain reaction to detect T-cell receptor- $\beta$ gene rearrangement was performed using DNA extracted from fresh or paraffin-embedded biopsies and the 'Biomed-2' primers and protocol (InVivoScribe Technologies, San Diego, CA, USA). ${ }^{25}$

\section{Statistical Analysis}

Fisher's exact test, Student's t-test and one-way ANOVA were performed to evaluate differences between datasets and a $P$-value $<0.05$ was considered significant.

\section{Results}

\section{Evaluation of Subepithelial Collagen}

Ten small bowel biopsies from consecutive, unselected adults with normal villous architecture (7 women, 3 men, mean age 63 years, range 23-87 years) who had reflux esophagitis $(n=3)$, reactive gastropathy $(n=5)$ and Helicobacter pylori-associated gastritis $(n=2)$, served as one set of controls. Trichrome-stained sections highlighted a thin, often discontinuous, basement membrane $<1.5 \mu \mathrm{m}$ in thickness (Figure 1a). As it has been proposed that active celiac disease patients frequently have subepithelial basement membrane thickening or fibrosis, ${ }^{4,11}$ trichrome-stained biopsies from 20 individuals with active celiac disease, 10 each with subtotal and total villous atrophy (15 women, 5 men, mean age 43 years, range 19-73 years) were evaluated. Subepithelial basement membrane thickness was similar to normal controls $(<1.5 \mu \mathrm{m})$ in 8 (40\%) (Figure 1b) and $12(60 \%)$ biopsies showed minimal fibrosis (mean $3 \pm 0.7 \mu \mathrm{m}$, range $1-5 \mu \mathrm{m}$ ) (Figure 1c). Thus, the 'minimal' inclusion (and diagnostic) criterion of collagenous sprue for this study was any case with a mean subepithelial collagen thickness exceeding $5 \mu \mathrm{m}(>$ mean +2 s.d. of active celiac disease controls).

The study cases were divided into three groups: (1) mild fibrosis ( $>5 \mu \mathrm{m}-10 \mu \mathrm{m}, n=6)$-splayed and multilayered collagen strands with entrapped capillaries (Figures 2a and b); (2) moderate fibrosis ( $>10 \mu \mathrm{m}-20 \mu \mathrm{m}, n=10$ )-collagen deposits of variable thickness with stellate extensions into the superficial lamina propria and along lateral portions of villi and crypts (Figures 2c and d); (3) marked fibrosis $(>20 \mu \mathrm{m}, n=3)$-dense, hyalinized subepithelial collagen bands with an amorphous appearance occupying $1 / 3$ to $1 / 2$ of the lamina propria (Figures 2e and f). The majority of cases (12/19, 
a

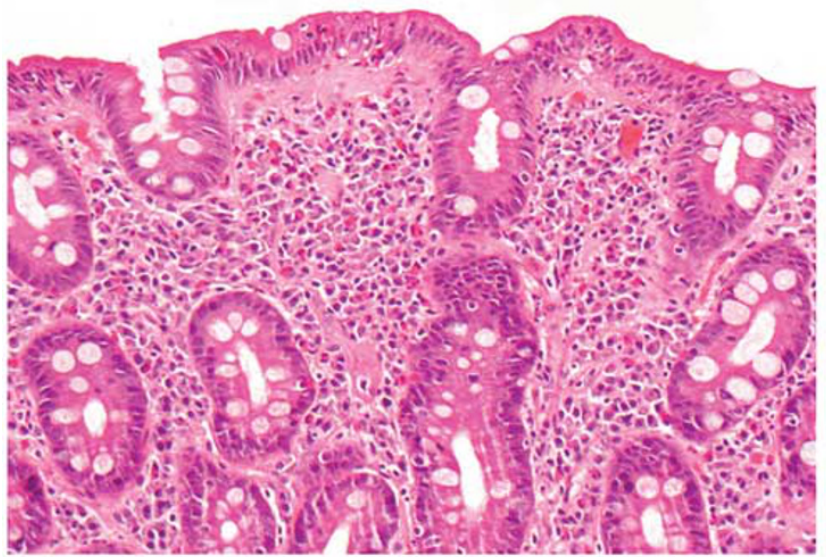

C

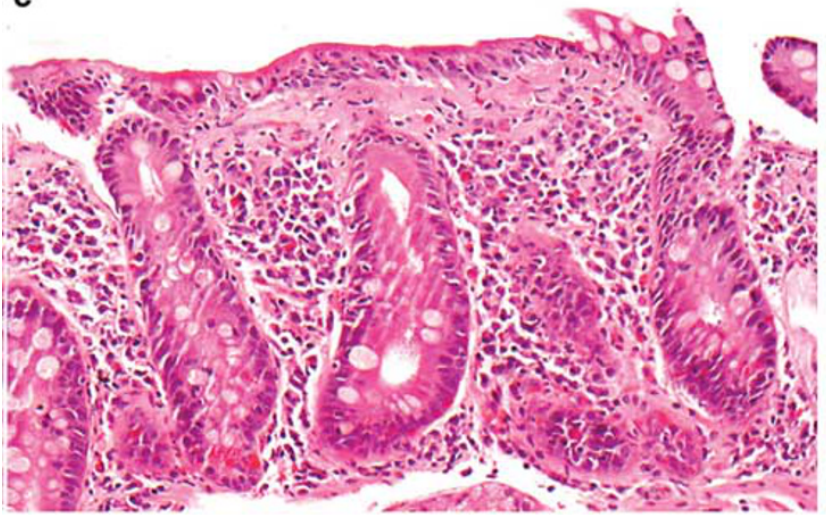

e

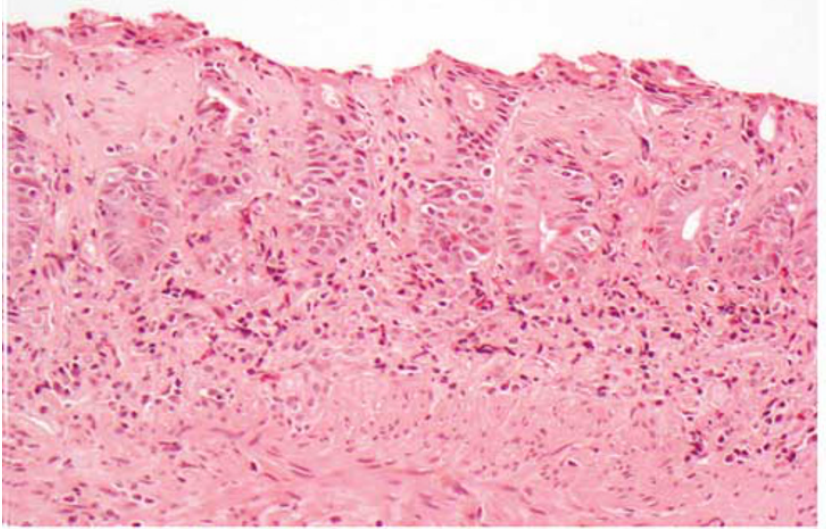

b

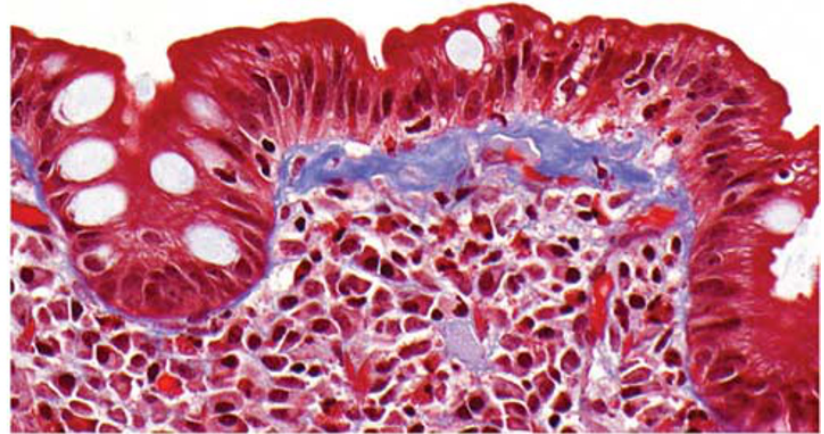

d

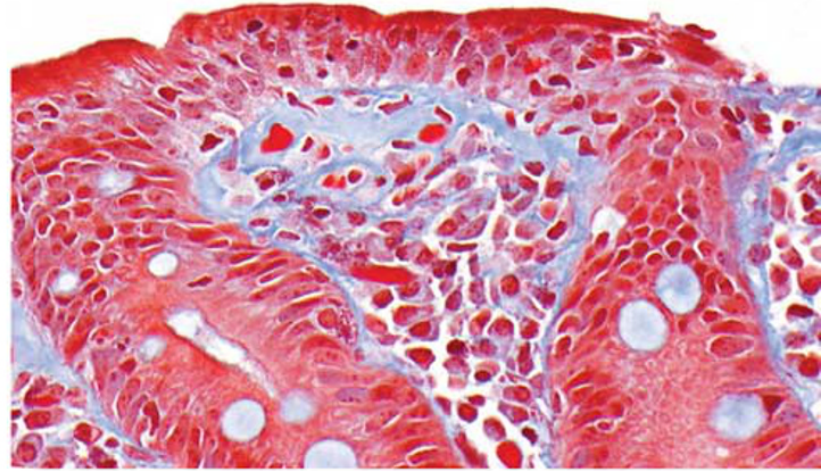

f

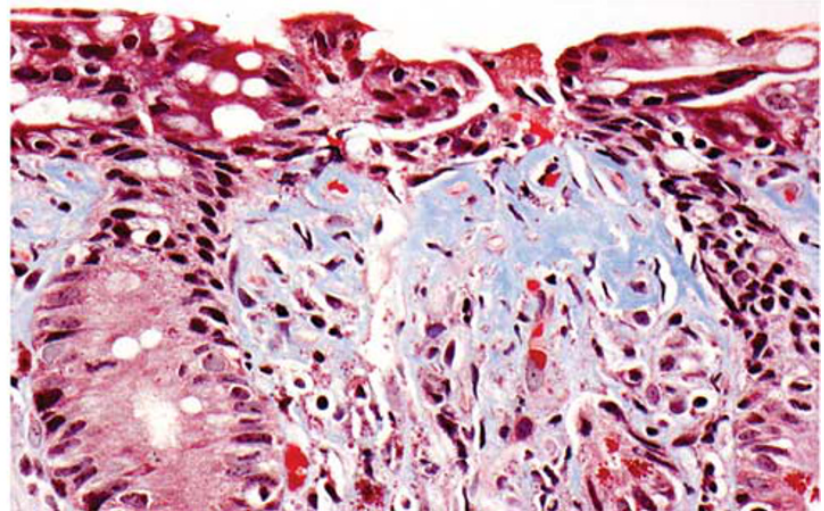

Figure 2 Representative examples of collagenous sprue cases with mild subepithelial fibrosis (case no. 5; (a), H\&E, $\times 200$; (b), Trichrome, $\times 400$ ), moderate fibrosis (case no. 16; (c), H\&E, $\times 200$; (d), Trichrome, $\times 400)$ and marked fibrosis (case no. 19; (e), H\&E, $\times 200 ;(\mathbf{f})$, Trichrome, $\times 400)$.

$63 \%$ ) across all grades of fibrosis had a patchy distribution of collagen (Table 2) and showed significant variability in the extent of fibrosis; nine $(75 \%)$ had at least one biopsy piece with diffuse fibrosis (and at least one piece that lacked appreciable fibrosis), two (17\%) showed fibrosis in all pieces, although some biopsy pieces only showed partial fibrosis, and one (8\%) case had an admixture of partially fibrotic and normal appearing pieces. All pieces with partial fibrosis showed fibrosis of at least $1 / 3$ of the length of the biopsy. 
Table 1 Clinical features of patients with collagenous sprue

\begin{tabular}{|c|c|c|c|c|c|c|c|c|c|c|c|c|c|c|}
\hline Patient & $\begin{array}{c}\text { Age } \\
\text { (years) }\end{array}$ & Sex & Serologies & $H L A-D Q$ & $\begin{array}{l}\text { Etiology } \\
\text { and clinical } \\
\text { presentation } \\
\text { at dx of CS }\end{array}$ & $\begin{array}{l}\text { Childhood } \\
\text { Dx }\end{array}$ & $\begin{array}{c}\text { Duration of } \\
\text { symptoms } \\
\text { before } d x \\
\text { (years) }\end{array}$ & $\begin{array}{c}\text { Interval } \\
\text { between } \\
\text { etiologic } \\
\text { dx and CS } \\
\text { (years) }\end{array}$ & $\begin{array}{l}\text { Type of } \\
\text { symptoms }\end{array}$ & $\begin{array}{l}\text { Response } \\
\text { to GFD }\end{array}$ & $\operatorname{IMRx}$ & $\begin{array}{l}\text { Response } \\
\text { to IMRx }\end{array}$ & $\begin{array}{l}\text { Outcome } \\
\text { (follow-up } \\
\text { duration) }\end{array}$ & $\begin{array}{l}\text { Other } \\
\text { autoimmune } \\
\text { or immune } \\
\text { disorders }\end{array}$ \\
\hline 1 & 54 & $\mathrm{~F}$ & IgA tTG+ & ND & $\begin{array}{l}\text { Primary } \\
\text { RCD (type I) }\end{array}$ & No & 10 & 3 & $\begin{array}{l}\text { Classical } \\
\text { (diarrhea) }\end{array}$ & No & Prednisone & Yes & A (4.3 years) & None \\
\hline 2 & 66 & $\mathrm{~F}$ & IgA tTG+ & DQ2/DQ8 & ACD & No & 10 & 1 & $\begin{array}{l}\text { Classical } \\
\text { (diarrhea) }\end{array}$ & No & Budesonide & Yes & $\mathrm{A}^{\mathrm{b}}(0.8$ years $)$ & $\begin{array}{l}\text { IgA } \\
\text { nephropathy }\end{array}$ \\
\hline 3 & 22 & $\mathrm{~F}$ & $\begin{array}{l}\text { IgGgliadin+, } \\
\text { IgA tTG+ }\end{array}$ & ND & ACD & Yes & 0 & 16 & $\begin{array}{l}\text { Atypical } \\
\text { (screening) }\end{array}$ & Yes & None & NA & A (7.5 years) & $\begin{array}{l}\text { DM type I, } \\
\text { peripheral } \\
\text { neuropathy }\end{array}$ \\
\hline 4 & 74 & $\mathrm{~F}$ & IgA tTG+ & ND & ACD & No & $\begin{array}{c}\text { Not } \\
\text { known }\end{array}$ & 0 & $\begin{array}{l}\text { Atypical } \\
\text { (anemia) }\end{array}$ & Yes & None & NA & A (2 years) & None \\
\hline 5 & 80 & $\mathrm{~F}$ & $\begin{array}{l}\text { IgA and } \\
\text { IgG gliadin+, } \\
\text { IgA tTG+ }\end{array}$ & ND & $\begin{array}{l}\text { Secondary } \\
\text { RCD (type I) }\end{array}$ & No & 2 & 4.4 & $\begin{array}{l}\text { Classical } \\
\text { (diarrhea) }\end{array}$ & Yes & None & NA & A (6.3 years) & $\begin{array}{l}\text { Dermatitis } \\
\text { herpetiformis }\end{array}$ \\
\hline 6 & 65 & $\mathrm{M}$ & $\begin{array}{l}\text { IgA gliadin+, } \\
\text { IgA tTG+ }\end{array}$ & ND & ACD & No & 10 & 0 & $\begin{array}{l}\text { Classical } \\
\text { (diarrhea) }\end{array}$ & Yes & None & NA & A (1.4 years) & None \\
\hline 7 & 34 & $\mathrm{~F}$ & IgA EMA+ & ND & ACD & No & 0.5 & 0 & $\begin{array}{l}\text { Classical } \\
\text { (diarrhea) }\end{array}$ & Yes & None & NA & A (6.8 years) & ANA 1:1280 \\
\hline 8 & 74 & $\mathrm{~F}$ & ND & ND & $\begin{array}{l}\text { Secondary } \\
\text { RCD (type I) }\end{array}$ & No & 8 & 12 & $\begin{array}{l}\text { Classical } \\
\text { (diarrhea) }\end{array}$ & No & $\begin{array}{l}\text { Prednisone } \\
\text { Azathioprine }\end{array}$ & Yes & LTF (0.3 year) & $\begin{array}{l}\text { Autoimmune } \\
\text { hepatitis }\end{array}$ \\
\hline 9 & 46 & $\mathrm{~F}$ & $\begin{array}{l}\text { IgA and } \\
\text { IgG gliadin+ }\end{array}$ & ND & $\begin{array}{l}\text { Secondary } \\
\text { RCD (type I) }\end{array}$ & Yes & 1 & 45 & $\begin{array}{l}\text { Classical } \\
\text { (diarrhea) }\end{array}$ & No & Prednisone & Yes & A (3.6 years) & $\begin{array}{l}\text { Autoimmune } \\
\text { hepatitis }\end{array}$ \\
\hline 10 & 82 & $\mathrm{~F}$ & $\mathrm{ND}$ & ND & $\begin{array}{l}\text { Secondary } \\
\text { RCD (type I) }\end{array}$ & No & 32 & 39 & $\begin{array}{l}\text { Classical } \\
\text { (diarrhea) }\end{array}$ & No & Azathioprine & Yes & LTF (0.4 year) & None \\
\hline 11 & 59 & $\mathrm{~F}$ & $\begin{array}{l}\text { IgA and } \\
\text { IgG gliadin+, } \\
\text { IgA EMA+ }\end{array}$ & ND & $\begin{array}{l}\text { Secondary } \\
\text { RCD (type I) }\end{array}$ & No & 20 & 5.3 & $\begin{array}{l}\text { Atypical } \\
\text { (anemia, } \\
\text { intermittent } \\
\text { diarrhea) }\end{array}$ & Yes & None & NA & A (5 years) & None \\
\hline 12 & 58 & $\mathrm{~F}$ & $\begin{array}{l}\text { IgA and } \\
\text { IgG gliadin-, } \\
\text { IgA tTG-, } \\
\text { IgA EMA-, } \\
\text { anti-enterocyte- }\end{array}$ & DQ2 & $\begin{array}{l}\text { Unclassified } \\
\text { sprue }\end{array}$ & No & 30 & 0 & $\begin{array}{l}\mathrm{NA}^{\mathrm{a}} \\
\text { (diarrhea) }\end{array}$ & No & $\begin{array}{l}\text { Prednisone } \\
\text { Budesonide }\end{array}$ & Yes & A (2 years) & $\begin{array}{l}\text { Hypogamma- } \\
\text { globulinemia }\end{array}$ \\
\hline 13 & 30 & $\mathrm{~F}$ & $\begin{array}{l}\text { IgA and } \\
\text { IgG gliadin+, } \\
\text { IgA tTG+ }\end{array}$ & ND & CD on GFD & Yes & 30 & 29 & $\begin{array}{l}\text { Classical } \\
\text { (diarrhea) }\end{array}$ & Yes & None & NA & A (3 years) & Psoriasis \\
\hline 14 & 63 & $\mathrm{~F}$ & $\begin{array}{l}\text { IgA gliadin+, } \\
\text { IgA tTG- } \\
\text { anti-enterocyte-- }\end{array}$ & ND & $\begin{array}{l}\text { Unclassified } \\
\text { sprue }\end{array}$ & No & 0.8 & 0 & $\begin{array}{l}\mathrm{NA}^{\mathrm{a}} \\
\text { (diarrhea) }\end{array}$ & No & Prednisone & Yes & A (0.9 years) & $\begin{array}{l}\text { Autoimmune } \\
\text { arthritis }\end{array}$ \\
\hline 15 & 65 & M & IgA tTG+ & ND & CD on GFD & Yes & 5 & 28 & $\begin{array}{l}\text { Classical } \\
\text { (diarrhea) }\end{array}$ & Yes & None & NA & A (0.4 years) & None \\
\hline 16 & 63 & $\mathrm{M}$ & IgA tTG+ & DQ2 & ACD & No & 0.5 & 1 & $\begin{array}{l}\text { Classical } \\
\text { (diarrhea) }\end{array}$ & No & Budesonide & Yes & $\mathrm{A}^{\mathrm{b}}(1.3$ years $)$ & $\begin{array}{l}\text { IgA } \\
\text { deficiency }\end{array}$ \\
\hline 17 & 69 & $\mathrm{~F}$ & ND & DQ2 & $\begin{array}{l}\text { Primary } \\
\text { RCD (type I) }\end{array}$ & No & 0.5 & 1.2 & $\begin{array}{l}\text { Atypical } \\
\text { (osteoporosis }\end{array}$ & No & $\begin{array}{l}\text { Prednisone } \\
\text { Azathioprine } \\
\text { Cyclosporine }\end{array}$ & Yes & $\mathrm{A}^{\mathrm{b}}$ (9.9 years) & $\begin{array}{l}\text { Peripheral } \\
\text { neuropathy } \\
\text { and dermatitis } \\
\text { herpetiformis }\end{array}$ \\
\hline
\end{tabular}




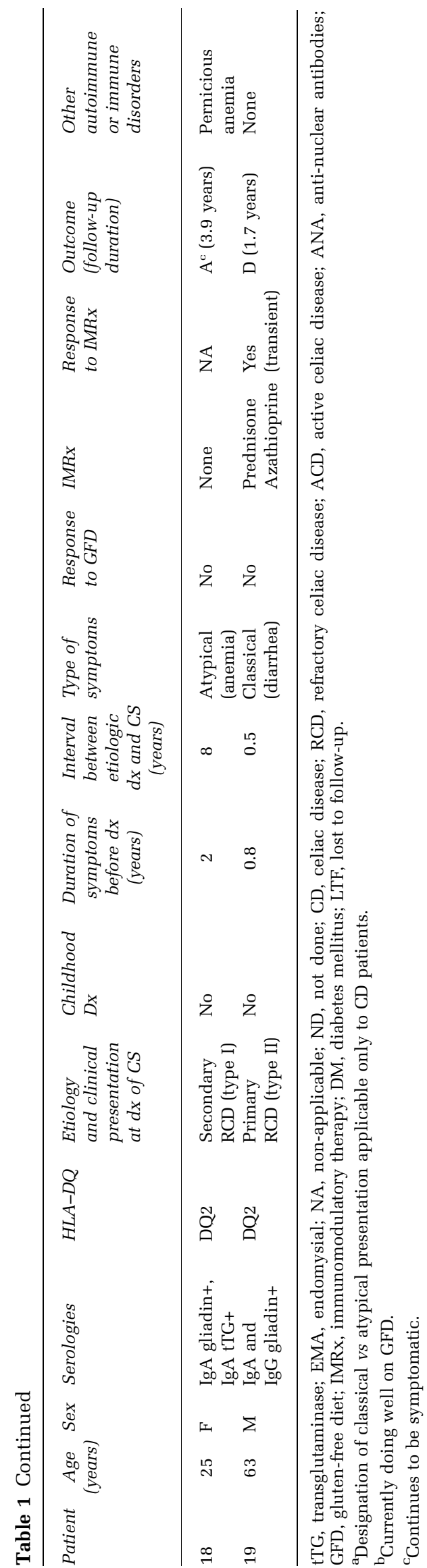

\section{Clinical Characteristics}

The clinical features of the 19 study patients (15 women, 4 men, mean age 57 years, range $22-80$ years) are summarized in Table 1. Seventeen of $19(89 \%)$ individuals had celiac disease, comprising 3\% (17/554) of all celiac disease patients who had biopsies reviewed at our center during the study period; 14 had positive serologies for celiac disease and three were diagnosed before serologies were routinely performed, based on histological findings and a response to gluten-free diet. Of these patients, nine (53\%) had refractory celiac disease at the time collagenous sprue was diagnosed (three primary, and six secondary refractory celiac disease, all except one had refractory celiac disease type I), six (35\%) had active celiac disease and two $(12 \%)$ were well controlled on gluten-free diet (endoscopy was performed for abdominal pain and dysphagia-biopsies revealed reactive gastropathy and esophageal ulceration, respectively). Of the 15 patients with refractory celiac disease or active celiac disease, $67 \%$ had classical and $33 \%$ had an atypical presentation (Table 1). Mean duration of celiac disease was 10.2 years (range 0-45 years). Four patients had an initial childhood diagnosis of celiac disease. Two of these patients (cases nos. 13, 15) had increased subepithelial collagen despite being well controlled on gluten-free diet at presentation, but they had a history of long periods of gluten ingestion before re-diagnosis of celiac disease as adults. Two patients (cases nos. 9, 15) showed severe villous atrophy, but no fibrosis on biopsy 5 years before their diagnostic biopsy.

In two $(11 \%)$ patients, designated as having unclassified sprue, an immune-mediated etiology of enteropathy was considered likely based on the atypical histological features (see section 'Histologic Findings') and presence of hypogammaglobulinemia in one and autoimmune arthritis in the other patient; celiac disease was excluded based on a combination of negative serologies and non response to gluten-free diet. Anti-enterocyte antibodies were not detected in these two patients.

All patients with available information $(n=6)$ were HLA-DQ2 + , including one who did not have celiac disease. Overall, $12(63 \%)$ patients had coexistent autoimmune or immune disorders (Table 1), 7/9 (78\%) patients had microscopic colitis, whereas lymphocytic gastritis $(n=2)$ and collagenous gastritis $(n=2)$ were detected in $4 / 16(25 \%)$ (Table 2). A diffuse process affecting the entire gastrointestinal tract (either intraepithelial lymphocytosis or subepithelial collagen deposition) was seen in two patients (case nos. 15, 18, Table 2). None of the patients had evidence of inflammatory bowel disease.

\section{Histologic Findings}

Total and subtotal villous atrophy was seen in 68 and $32 \%$ of cases, respectively (Table 2). Surface epithelial damage was seen in all cases and detach- 
Table 2 Collagenous sprue: endoscopic and pathologic findings

\begin{tabular}{|c|c|c|c|c|c|c|c|c|c|c|c|c|c|c|c|c|c|}
\hline & & & & SE colld & agen thickn & & Distı & ribution of & f collagen & & Neut & trophils & & & & & \\
\hline & tient & Endoscopic & Degree of & Category & Range & Avg & Overall & Affected & Distribution & $L P$ & Present & Location & No. & No. of IELs/ & Percentage & $P C R$ & Other GI \\
\hline 1 & $\mathrm{dx} b \mathrm{bx}$ & Not known & Total & Mild & $7.6-11$ & 8.7 & Patchy & $3 / 4$ & $2 \mathrm{D}, 1 \mathrm{P}$ & Moderate & Yes & LP, SE & 4 & 35 (mild) & 78 & Polyclonal & $\begin{array}{l}\text { Collagenous } \\
\text { colitis }\end{array}$ \\
\hline & f/u (1 year) & Not known & Subtotal & Mild & $8.5-10.2$ & 9.1 & Patchy & $2 / 4$ & $2 \mathrm{D}$ & Moderate & Yes & LP, SE & 5 & 38 (mild) & 63 & Polyclonal & \\
\hline & f/u (3 years) & Not known & Total & Mild & $8.5-10.2$ & 9.1 & Patchy & $3 / 5$ & $1 \mathrm{D}, 2 \mathrm{P}$ & Mild & Yes & LP, SE & 7 & 37 (mild) & 54 & Polyclonal & \\
\hline 2 & $d x$ bx & NOD, SC & Subtotal & Mild & $7.6-9.3$ & 8.7 & Patchy & $4 / 7$ & $4 \mathrm{P}$ & Moderate & Yes & LP, SE, CE & 11 & 92 (marked) & 82 & Polyclonal & $\begin{array}{l}\text { Lymphocytic } \\
\text { colitis }\end{array}$ \\
\hline 3 & $\mathrm{dx} b \mathrm{bx}$ & MP, AF, SC & Subtotal & Mild & $8.5-10.2$ & 9.3 & Diffuse & $3 / 3$ & $3 \mathrm{D}$ & Moderate & No & NA & 13 & 32 (mild) & 60 & Polyclonal & \\
\hline & f/u (1 year) & MP, RF, SC & Partial & Wnl & $1.7-3.4$ & 2.3 & NA & NA & NA & Moderate & No & NA & 20 & 62 (moderate) & 65 & Polyclonal & \\
\hline 4 & $d x b x$ & Not known & Subtotal & Mild & $8.5-11$ & 9.6 & Patchy & $3 / 3$ & $1 \mathrm{D}, 2 \mathrm{P}$ & Marked & Yes & LP, SE & 17 & 69 (moderate) & 81 & Polyclonal & \\
\hline 5 & $\mathrm{dx} b \mathrm{bx}$ & $\mathrm{MP}, \mathrm{AF}$ & Total & Mild & $9-10.2$ & 9.8 & Diffuse & $6 / 6$ & $6 \mathrm{D}$ & Marked & Yes & LP & 30 & 32 (mild) & 72 & Minor clone & \\
\hline & f/u (4 year) & NOD, AF & Total & Mild & $7.8-11$ & 9.6 & Diffuse & $9 / 9$ & $9 \mathrm{D}$ & Moderate & Yes & LP, SE & 32 & 43 (mild) & 75 & Polyclonal & \\
\hline 6 & $d x b x$ & SC & Total & Mild & $9-10.2$ & 9.8 & Patchy & $3 / 4$ & $2 \mathrm{D}, 1 \mathrm{P}$ & Moderate & Yes & LP & 20 & 82 (marked) & 48 & Polyclonal & \\
\hline & f/u (1 year) & SC & Partial & Mild & $5.4-8.5$ & 7.5 & Patchy & $3 / 8$ & $3 \mathrm{P}$ & Mild & Yes & LP & 32 & 73 (moderate) & 45 & Polyclonal & \\
\hline 7 & $\mathrm{dx} b \mathrm{bx}$ & SC & Total & Moderate & $10.2-11.7$ & 11.2 & Patchy & $2 / 4$ & $2 \mathrm{D}$ & Moderate & Yes & LP & 35 & 32 (mild) & 40 & Polyclonal & $\begin{array}{l}\text { Lymphocytic } \\
\text { colitis }\end{array}$ \\
\hline & f/u (1 year) & $\mathrm{RF}$ & Partial & Wnl & $1.7-3.4$ & 2.3 & NA & NA & NA & Moderate & No & NA & 8 & 12 (absent) & 33 & Polyclonal & \\
\hline 8 & $\mathrm{dx}$ bx & MP & Total & Moderate & $9.6-12.7$ & 11.4 & Diffuse & $5 / 5$ & $5 \mathrm{D}$ & Moderate & No & NA & 40 & 72 (moderate) & 28 & Minor clone & $\begin{array}{l}\text { Lymphocytic } \\
\text { colitis }\end{array}$ \\
\hline & f/u (3 months) & Not known & Subtotal & Wnl & $3.4-7.6$ & 4.3 & NA & NA & NA & Moderate & No & NA & 9 & 52 (moderate) & 47 & Polyclonal & \\
\hline 9 & $d x b^{a}$ & MP, NOD & Total & Moderate & $11-12.7$ & 12.1 & Diffuse & $10 / 10$ & $10 \mathrm{D}$ & Marked & Yes & LP, SE, CE & 38 & 38 (mild) & 40 & Polyclonal & \\
\hline 10 & $\mathrm{dx} b x$ & $\mathrm{AF}, \mathrm{MP}$ & Subtotal & Moderate & $9-15$ & 12.3 & Patchy & $3 / 5$ & $1 \mathrm{D}, 2 \mathrm{P}$ & Moderate & No & NA & 7 & 55 (moderate) & 50 & Polyclonal & $\begin{array}{l}\text { Collagenous } \\
\text { colitis }\end{array}$ \\
\hline & f/u (5 months) & $\mathrm{AF}, \mathrm{MP}, \mathrm{SC}$ & Partial & Wnl & $1.7-7.6$ & 4.1 & NA & NA & NA & Moderate & Yes & LP & 25 & 43 (mild) & 55 & Polyclonal & \\
\hline 11 & $\mathrm{dx} b x$ & $\mathrm{AF}$ & Subtotal & Moderate & $10.2-13.7$ & 12.6 & Patchy & $4 / 6$ & $2 \mathrm{D}, 2 \mathrm{P}$ & Marked & No & NA & 40 & 58 (moderate) & 40 & Polyclonal & \\
\hline 12 & $d x$ bx & NOD & Total & Moderate & 13.6-17 & 15.8 & Patchy & $5 / 7$ & $4 \mathrm{D}, 1 \mathrm{P}$ & Moderate & Yes & LP, SE, CE & 15 & 15 (absent) & 80 & Polyclonal & $\begin{array}{l}\text { Collagenous } \\
\text { gastritis }\end{array}$ \\
\hline & f/u (4 months) & Not known & Subtotal & Mild & $3.4-11.9$ & 6.2 & Patchy & $1 / 3$ & $1 \mathrm{P}$ & Moderate & Yes & LP & 7 & 8 (absent) & ND & ND & \\
\hline & f/u (1 year) & Not known & Partial & Wnl & $1.0-5.1$ & 3.3 & NA & NA & NA & Mild & Yes & LP & 8 & 10 (absent) & ND & ND & \\
\hline & f/u (1.5 year) & Not known & Normal & Normal & $1.0-1.5$ & $<1.5$ & NA & NA & NA & Mild & No & NA & 7 & 6 (absent) & ND & ND & \\
\hline 13 & $d x b x$ & SC & Total & Moderate & $14.3-19.2$ & 16.7 & Diffuse & $3 / 3$ & $3 \mathrm{D}$ & Moderate & Yes & LP & 50 & 20 (absent) & 90 & Polyclonal & \\
\hline 14 & $\mathrm{dx} b \mathrm{bx}$ & $\mathrm{AF}$ & Total & Moderate & $13.6-15.3$ & 14.1 & Diffuse & $3 / 3$ & $3 \mathrm{D}$ & Marked & Yes & LP, SE, CE & 19 & 8 (absent) & 80 & $\mathrm{ND}$ & \\
\hline 15 & $d x b x^{a}$ & $\mathrm{AF}$ & Total & Moderate & $12-15.6$ & 13.7 & Patchy & $3 / 4$ & $2 \mathrm{D}, 1 \mathrm{~F}$ & Marked & No & NA & 52 & 25 (absent) & 88 & Polyclonal & $\begin{array}{l}\text { Lymphocytic } \\
\text { gastritis } \\
\text { Lymphocytic } \\
\text { colitis }\end{array}$ \\
\hline 16 & $\mathrm{dx} b \mathrm{bx}$ & NOD, AF & Subtotal & Moderate & $16.2-19.4$ & 18 & Diffuse & $9 / 9$ & $9 \mathrm{D}$ & Moderate & Yes & LP & 29 & 70 (moderate) & 87 & Polyclonal & \\
\hline & f/u (1 year) & $\mathrm{AF}$ & Partial & Wnl & $1.0-3.4$ & 2.2 & NA & NA & NA & Moderate & No & NA & 15 & 20 (absent) & 80 & ND & \\
\hline 17 & $\mathrm{dx} b x$ & MP, NOD & Total & Marked & $20-24$ & 21.3 & Patchy & $2 / 3$ & $2 \mathrm{D}$ & Marked & Yes & LP, SE, CE & 23 & 42 (mild) & 76 & Polyclonal & \\
\hline & f/u (2 year) & MP, RF & Subtotal & Mild & $8.5-10.2$ & 9.6 & Patchy & $1 / 5$ & $1 \mathrm{P}$ & Moderate & Yes & LP, SE & 56 & 8 (absent) & 62 & ND & \\
\hline 18 & $\mathrm{dx} b \mathrm{x}$ & NOD & Total & Marked & $22-28$ & 24 & Patchy & $4 / 5$ & $4 \mathrm{D}$ & Marked & No & NA & 61 & 62 (moderate) & 92 & Polyclonal & $\begin{array}{l}\text { Collagenous } \\
\text { gastritis } \\
\text { Collagenous } \\
\text { colitis }\end{array}$ \\
\hline
\end{tabular}




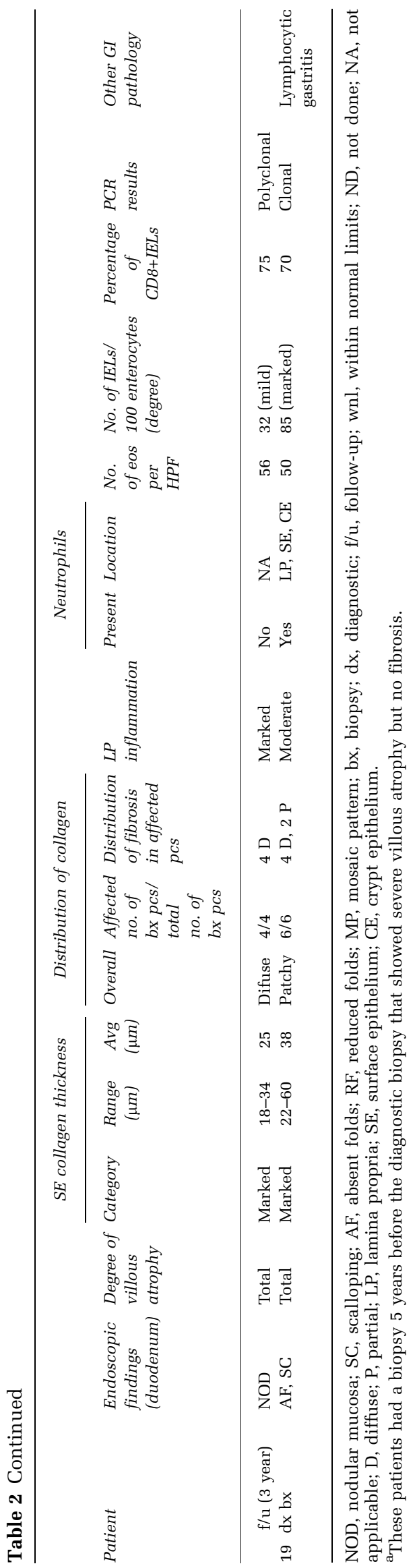

ment of the surface epithelium was observed in the majority (89\%). Neutrophils were observed in $68 \%$ of cases, but no association between the presence or location of neutrophils and the degree of lamina propria inflammation or fibrosis was noted. The average number of eosinophils per high power field was significantly higher in cases with moderate and marked fibrosis compared to those with mild fibrosis (32.5 and 44.7 vs 15.8, $P=0.04$ ), although no association with the extent of lamina propria inflammation was observed. An immunohistochemical stain for smooth muscle antigen did not show any increase in the number of myofibroblasts in the subepithelial zone in any group (data not shown).

One case of unclassified sprue (case no. 12, Figures 3a and b) showed total villous atrophy, surface epithelial damage, moderate chronic inflammation of the lamina propria and distension by prominent lymphoid follicles with reactive germinal centers (nodular lymphoid hyperplasia), neutrophilic infiltrate, and cryptitis. The second case of unclassified sprue (case no. 14, Figures 3c-f)) showed total villous atrophy with crypt atrophy and loss, extensive pericryptal fibrosis, and cryptitis, scattered crypts had apoptotic bodies. The inflammatory infiltrate consisted of lymphocytes, plasma cells, neutrophils, eosinophils, and histiocytes. Granulomas were seen in association with degenerating crypts and mucin extravasation, which was especially prominent in Brunner's glands. Stains for bacterial, fungal, and viral organisms were negative in both cases.

\section{Evaluation of Intraepithelial Lymphocytes}

Fifteen of $17(88 \%)$ celiac disease cases showed increased intraepithelial lymphocytes, but the two cases of unclassified sprue did not. The degree of intraepithelial lymphocytosis and the percentage of CD8 + intraepithelial lymphocytes in all cases evaluated are described in Table 2. The mean number of intraepithelial lymphocytes did not differ significantly between cases with mild, moderate and marked fibrosis $(57,38,63, P=0.2)$. A significant expansion of CD3 + CD8- intraepithelial lymphocytes $(50 \%)$ was restricted to cases with mild $(n=1)$ or moderate $(n=5)$ fibrosis (Figure 4$)$.

Flow cytometry was performed in four cases, including three of six with significant CD3 + CD8intraepithelial expansions. In all cases, the CD8intraepithelial lymphocytes corresponded to expansions of T-cell receptor- $\gamma \delta+$ lymphocytes (range 22$43 \%$ of all gated cells). Intraepithelial lymphocytes with aberrant phenotype, namely concurrent CD8 loss and surface CD3 and/or T-cell receptor downregulation, were not detected.

Polymerase chain reaction for T-cell receptor- $\beta$ gene rearrangement showed polyclonal products in $15 / 18(83 \%)$ cases tested (Table 2). Minor clones, in a polyclonal background, seen in two cases were not 


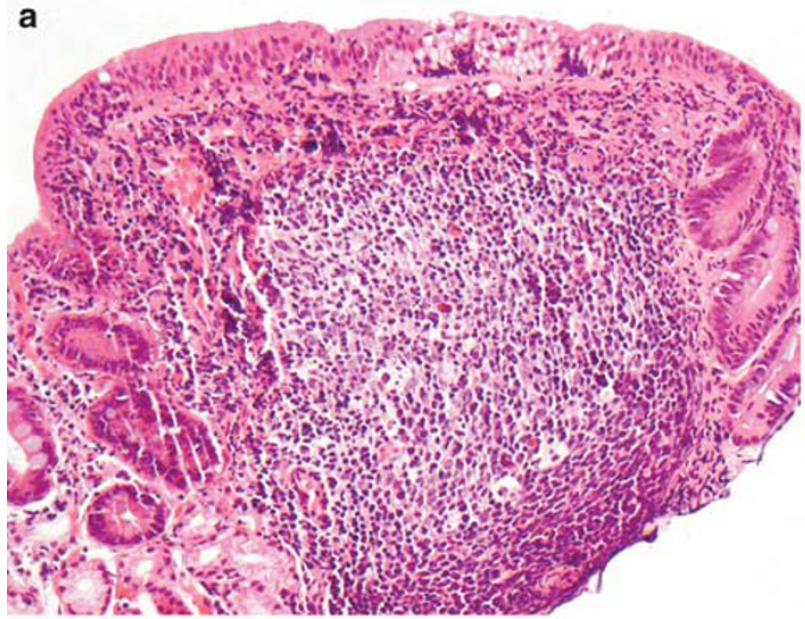

b

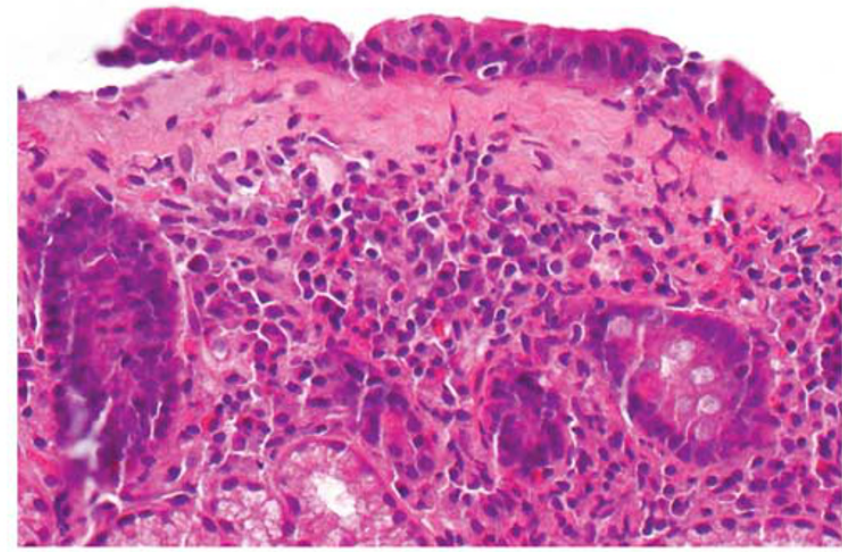

C
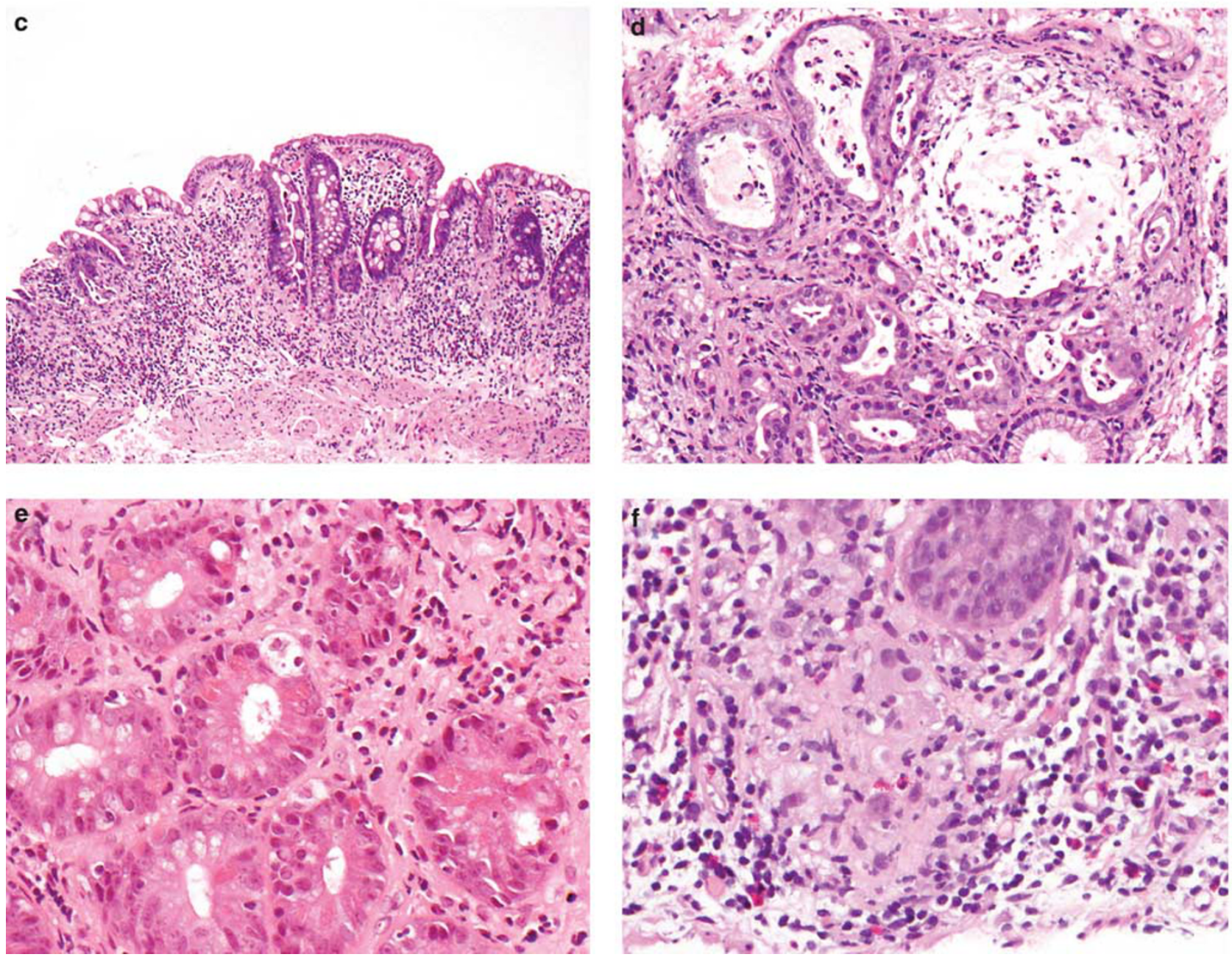

Figure 3 Histological features of unclassified sprue (H\&E). Prominent lymphoid follicles indicating nodular lymphoid hyperplasia $(\mathbf{a}, \times 100)$ and subepithelial fibrosis, but an absence of intraepithelial lymphocytosis $(\mathbf{b}, \times 200)$ in case no. 12 . Crypt atrophy and loss $(\mathbf{c}, \times 100)$, degenerating Brunner's glands and mucin extravasation $(\mathbf{d}, \times 200)$, crypt with apoptotic cells $(\mathbf{e}, \times 400)$ and pericryptal granulomas (f, $\times 400)$, in addition to subepithelial fibrosis, were present in case no. 14 .

detected in follow-up biopsies, suggesting the presence of transient T-cell expansions with skewed T-cell receptor repertoires either in the epithelial compartment or the lamina propria. These cases did not meet the criteria for refractory celiac disease type II. ${ }^{5}$ A dominant clone was detected in the small bowel biopsy in only one case. The intraepithelial lymphocytes (CD3 + CD8 + ) lacked an aberrant 


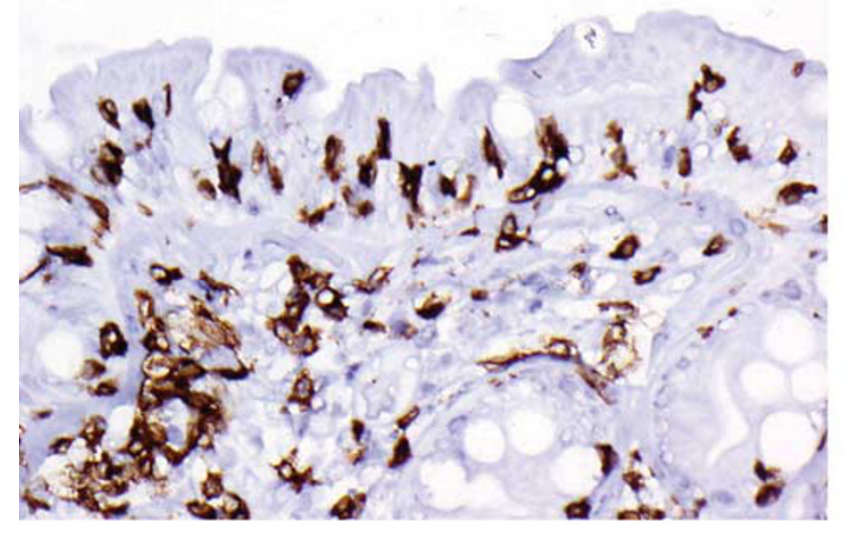

C

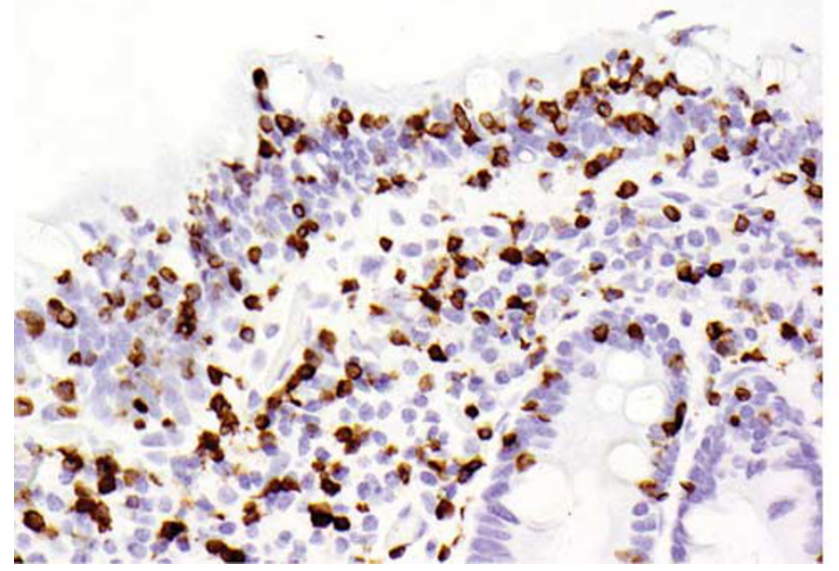

b

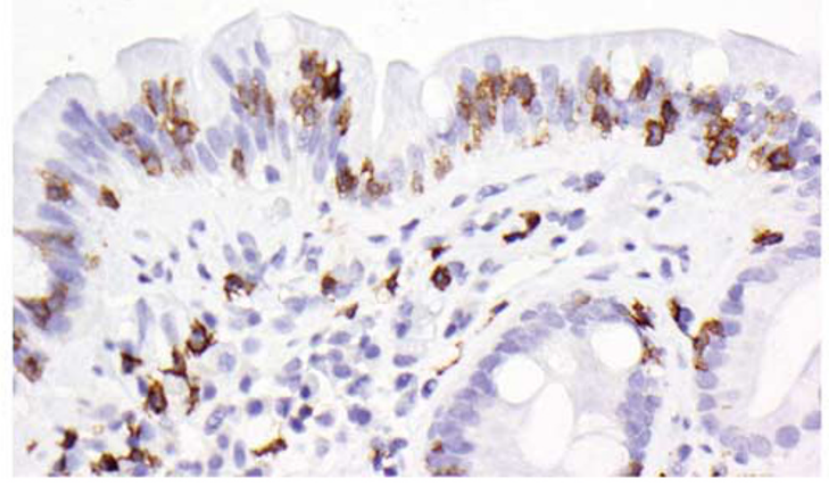

d

Figure 4 Representative cases of collagenous sprue showing expansions of CD3 + CD8 + IELs (case no. 4; (a) CD3, (b) CD8; $\times 400)$ and CD3 + CD8- IELs (case no. 8, (c) CD3, (d) CD8; × 400).

phenotype by immunohistochemical staining; however, as flow cytometry was not performed in this case, surface CD3 expression could not be evaluated. This case was classified as refractory celiac disease type II, as rare cases of refractory celiac disease type II with similar phenotype have been described. ${ }^{24,26}$ A clonal product of identical size was also detected in the lung of this patient, but not in peripheral blood.

\section{Association of Subepithelial Fibrosis with Disease Presentation, Response to Treatment and Outcome}

The clinical presentation of patients with mild fibrosis was refractory $(n=2)$ and active $(n=4)$ celiac disease, whereas moderate thickening was observed in individuals with refractory $(n=4)$ and active celiac disease $(n=2)$ and those on gluten-free diet $(n=2)$ or with unclassified sprue $(n=2)$. All three individuals with marked thickening had refractory celiac disease. Atypical presentation of celiac disease was seen in patients across all grades of fibrosis. There was no association between a diarrhea predominant presentation and the degree of fibrosis (4/6 with mild fibrosis vs 10/13 with moderate/marked fibrosis, $P=0.4$ ). Similarly, frequencies of coexistent autoimmune/immune disorders or other gastrointestinal pathology did not differ significantly between patients with mild fibrosis and those with moderate/marked fibrosis (3/6 vs $9 / 13, P=0.6$ and $2 / 6$ vs $7 / 13, P=0.6$, respectively).

Eight of 17 (47\%) celiac disease patients did well on gluten-free diet; four with mild and four with moderate fibrosis. Two of the latter had secondary refractory celiac disease type I. Response to glutenfree diet was seen more frequently in patients with mild compared to those with moderate/marked fibrosis, although the difference was not statistically significant (4/6 vs 4/13, $P=0.3$ ). Similarly, though not statistically significant, patients who responded to gluten-free diet had a lower frequency of autoimmune/immune conditions and other gastrointestinal pathology compared with those who did not $(4 / 8$ vs $8 / 11, P=0.4,2 / 8$ vs $7 / 11, P=0.2)$.

Three patients required parenteral nutrition. Ten patients, across all grades of fibrosis, received 

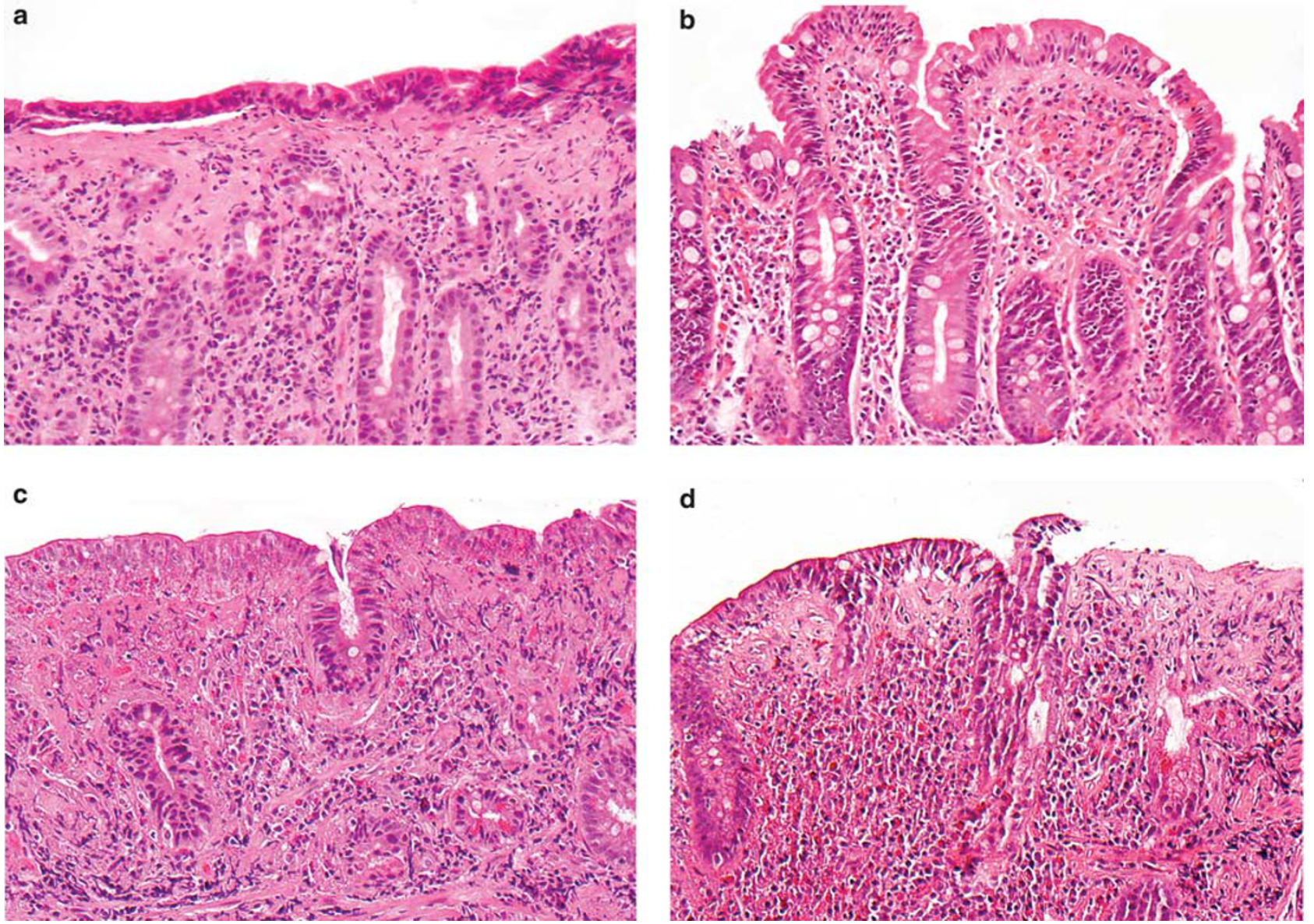

Figure 5 Diagnostic and follow-up small bowel biopsies (H\&E): case no. 17 shows initial total villous atrophy and marked subepithelial fibrosis $(\mathbf{a}, \times 200)$, subsequently showing subtotal villous atrophy and mild fibrosis $(\mathbf{b}, \times 200)$; case no. 18 shows total villous atrophy and marked fibrosis $(\mathbf{c}, \times 200)$ without improvement 3 years later $(\mathbf{d}, \times 200)$.

immunomodulatory therapy. A response to immunomodulatory therapy was seen in all patients across different clinical presentations (Table 1). Response was transient in the patient with refractory celiac diseased type II. One patient (case no. 17) with refractory celiac disease type I initially required immunomodulatory therapy, but is currently doing well on a gluten-free diet, whereas one patient (case no. 18) with refractory celiac disease type I refused immmunomodulatory therapy and has persistent iron-deficiency anemia on a gluten-free diet.

Sixteen of $19(84 \%)$ patients are currently alive (mean follow-up of 3.7 years, range 0.4-9.9 years); two were lost to follow-up after 3 and 5 months. The patient with refractory celiac disease type II died from complications of malnutrition 1.7 years after diagnosis of collagenous sprue. No patient developed lymphoma.

\section{Endoscopy and Colonoscopy Findings}

The endoscopic appearance of the duodenal mucosa was abnormal at diagnosis of collagenous sprue in
17 patients with available information (Table 2). Mucosal abnormalities persisted in all eight patients who had follow-up endoscopy, with qualitative changes seen in $6 / 8(75 \%)$ cases. Despite persistent endoscopic abnormalities, 6/8 (75\%) and 5/8 (63\%) cases showed improvement of villous architecture and reduction in fibrosis, respectively (see section 'Follow-Up Biopsies'). These findings are indicative of slow and possible incomplete (patchy) recovery, which is not detectable on endoscopy and parallel observations in uncomplicated celiac disease, where endoscopy has been shown to have low sensitivity in predicting histological alterations $(50-80 \%){ }^{27}$ None of the patients had ulcerations or erosions at diagnosis or follow-up. The colon had an unremarkable appearance in all nine cases evaluated.

\section{Follow-Up Biopsies}

A reduction in subepithelial collagen was seen in 7 of $11(64 \%)$ patients with follow-up small bowel biopsies (0.3-3 years post diagnosis of collagenous sprue) across all grades of fibrosis (Table 2, Figures $5 \mathrm{a}$ and b), the thickness reaching active celiac 
disease control range in 5, with 1 case each showing showing mild or no fibrosis. Improvement was seen in two patients on gluten-free diet and five treated with immunomodulatory therapy. Decreased fibrosis was accompanied by decreased degrees of villous atrophy in all cases. Persistent fibrosis was seen in four patients (Table 2, Figures 5c and d). One patient received immunomodulatory therapy, whereas three were on gluten-free diet. The degree of villous atrophy improved in two and no change was observed in two cases. Intraepithelial lymphocyte numbers were quite variable in both situations and did not appear to correlate with histological improvement (Table 2).

\section{Discussion}

Collagenous sprue has historically been considered a syndrome of recalcitrant malabsorption associated with high morbidity and mortality. ${ }^{2,11}$ This view has been challenged by a few. ${ }^{4,8}$ However, the clinical and pathological manifestations of this disorder have not been investigated adequately, as data regarding its prevalence and etiology are limited and definitional criteria are suboptimal. Our study represents the first large comprehensive single institution study describing the histopathological and phenotypical features, clinical characteristics, therapeutic strategies, and outcomes of individuals with a thickened small bowel subepithelial collagen table.

Qualitative descriptions of increased subepithelial fibrosis have been reported in $36-42 \%$ of small bowel biopsies from celiac disease patients, ${ }^{4,28}$ which have been interpreted as common and nonspecific findings lacking any clinical significance. ${ }^{4}$ It has been argued that a diagnosis of collagenous sprue requires the presence of 'a clear-cut layer of subepithelial collagen that extends into the lamina propria."11 Still others have based the diagnosis of collagenous sprue on the presence of $>10 \mu \mathrm{m}$ thickness of the subepithelial collagen band, using criteria proposed for the diagnosis of collagenous colitis. ${ }^{5}$ Morphometric analysis of the subepithelial collagen layer in small bowel biopsies from patients with active celiac disease, or for that matter other small intestinal diseases, has not been described. Hence, there is a lack of consensus as to what represents a 'significant' increase in subepithelial collagen, hampering the promulgation of histological criteria required for diagnosing collagenous sprue.

Our morphometric analysis was conservative avoiding areas with tangential orientation, which can lead to an erroneous impression of increased subepithelial collagen. ${ }^{29}$ Mild thickening of the basement membrane, not exceeding $5 \mu \mathrm{m}$, was seen in $60 \%$ of active celiac disease control patients who had severe villous atrophy, confirming previous reports. $^{4,28}$ Hence, we suggest that only cases with small bowel subepithelial collagen thickness $>5 \mu \mathrm{m}$ (approximately the diameter of a lymphocyte) should be diagnosed as collagenous sprue and recommend that a note indicating this finding (and if possible the degree) be provided in the histopathology report. This would alert the physician to the possibility of an atypical disease course.

Histological improvement was seen in the majority of our patients with available follow-up biopsies, irrespective of disease presentation and symptoms and across all grades of fibrosis, though without any identifiable predictors of response. Whether these findings represent true histological improvement or sampling artifacts can be debated. The concurrent improvement in mucosal architecture with a decrease in the extent of fibrosis and amelioration of clinical symptoms of our patients, supports the view of previous investigators, who documented the disappearance of collagen deposits on follow-up biopsies and suggested that collagenous sprue might be reversible in certain instances. ${ }^{4,8-10}$ In this context, it is worth reiterating that subepithelial collagen fibrosis was patchy in the majority of our cases, both at diagnosis and at follow up, with the extent and severity varying from piece to piece, as well as within a single biopsy piece. This observation is in keeping with the original description of collagenous sprue ${ }^{2}$ and mirrors the variability of mucosal architectural abnormalities in uncomplicated celiac disease, ${ }^{30} \mathrm{We}$, thus, recommend obtaining multiple biopsies from different regions of the proximal small bowel (altogether 4-6 pieces) to ensure adequate sampling, as has been suggested for celiac disease. ${ }^{31}$

Our study and previous reports indicate that most patients with collagenous sprue are middle-aged to elderly females. ${ }^{5,11}$ In our series, $21 \%$ of the individuals were younger than 35 years, highlighting the fact that young adults may occasionally be affected. ${ }^{32}$ However, in contrast to collagenous gastritis, ${ }^{33,34}$ collagenous sprue is rare in children, with only two cases described in the literature. ${ }^{1,13}$ The most common etiology of collagenous sprue in our patients was celiac disease, accounting for $89 \%$ of all collagenous sprue cases. The prevalence of collagenous sprue in this patient population was quite low (3\%), despite the inherent referral bias in our study. Thus, it is likely that the true prevalence of collagenous sprue among celiac disease patients in the community is lower. The relationship between celiac disease and collagenous sprue has remained controversial over the past several decades as many of the reported patients failed to respond to a gluten-free diet, ${ }^{3,8,10,11,32}$ and most cases lacked serologic or other clinical data that could have suggested celiac disease as the etiology. ${ }^{3,8,10,11}$ Nonetheless, Bossart et $a l^{4}$ observed 'moderate to marked' subepithelial collagen in $8 \%$ of their celiac disease patients. In two recent studies of refractory sprue, collagenous sprue accounted for 50 and $34 \%$ of all cases, the underlying etiology of collagenous sprue 
being refractory celiac disease in 40 and $86 \%$ of the cases, respectively. ${ }^{5,11}$

Collagenous sprue can also be a manifestation of other disorders. Eleven percent of our patients did not have celiac disease. Although the precise etiology could not be determined conclusively, immune-mediated mechanisms appeared likely. These patients did not respond to a gluten-free diet, but are currently symptom free on maintenance steroids. Small bowel biopsies from these patients had atypical histological features and lacked intraepithelial lymphocytosis. The latter may help distinguish cases of collagenous sprue unrelated to celiac disease. However, this feature should be interpreted in the context of other clinical, serological and histological variables, as patients on gluten-free diet often lack increased intraepithelial lymphocytes. ${ }^{35}$ Conversely, before attributing increased intraepithelial lymphocytes to underlying celiac disease, other mimics of celiac disease, which have also been implicated as etiologic agents of collagenous sprue, such as tropical sprue, common variable immunodeficiency and autoimmune enteropathy, should be excluded. ${ }^{17,18,36}$ Unusual histological features observed in our patients with unclassified sprue, included a peculiar pattern of crypt destruction, reminiscent of graft-versus-host disease, accompanied by a granulomatous reaction and nodular lymphoid hyperplasia, which can also be seen in small bowel biopsies from individuals with common variable immunodeficiency. ${ }^{17}$

An increased prevalence (63\%) of coexisting autoimmune conditions was noted in our patients, which is higher than the prevalence reported in celiac disease patients $(5-30 \%){ }^{37,38}$ This might explain the more pronounced skew in gender distribution, that is, female predominance observed in our series. An association with microscopic colitis, lymphocytic gastritis and/or collagenous gastritis was also seen, the former being more common and occurring at a higher frequency compared with celiac disease patients in general. ${ }^{39}$

A notable finding of our study, from a clinical perspective, was the significant frequency (33\%) of atypical or silent presentation of celiac disease patients who had collagenous sprue, which contrasts with the prevailing view. Atypical presentations are being increasingly recognized in celiac disease patients, ${ }^{40}$ likely because of a combination of more widespread use of serological testing and the changing nature of celiac disease. Furthermore, although the majority of our patients had refractory celiac disease, collagenous sprue was seen in patients with active celiac disease, confirming previous case reports, ${ }^{9,10,15}$ and interestingly even in asymptomatic individuals on gluten-free diet, which has not been previously reported. A history of celiac disease since childhood with long periods of gluten ingestion and the documentation of subepithelial fibrosis developing in follow up biopsies raise the possibility that duration of disease could be a factor in disease pathogenesis.
Our patient profile also differed from a previous study of refractory celiac disease type II, which described collagenous sprue in $86 \%$ of patients ${ }^{5}$ and the few individuals with collagenous sprue in the study of Daum et al. ${ }^{26}$ All but one of our patients with refractory celiac disease had type I disease, and in all cases evaluated, increased CD8- intraepithelial T-cells represented expansions of $\gamma \delta+$ T-cell receptor-bearing lymphocytes. The only patient with refractory celiac disease type II had CD8 + intraepithelial lymphocytes, clonal T-cell receptor- $\beta$ rearrangement and evidence of extraintestinal dissemination of the T-cell clone. ${ }^{26,41}$ Refractory celiac disease type II patients with CD8 + intraepithelial lymphocytes were not observed by Cellier et $a l,{ }^{5}$ but rare cases of clonal CD8 + intraepithelial lymphocyte expansions have been reported. ${ }^{24,26}$ Our patient with refractory celiac disease type II was the only one documented to have died. Further studies are needed to ascertain whether the incidence of refractory celiac disease type II is higher in collagenous sprue patients with fatal outcomes.

Patients who did not respond to gluten-free diet were more likely to have moderate or marked fibrosis, but only $62 \%$ of patients in both groups combined had refractory celiac disease. Of interest, $38 \%$ of these patients had refractory 'atypical' symptoms rather than diarrhea. It is important to note that patients with severe fibrosis also had a higher prevalence of autoimmune conditions and colonic or gastric pathology. These observations suggest that refractory symptoms and the need for immunomodulatory therapy might reflect a heavier or more complex disease burden or generalized immune deregulation and that collagen deposition might represent an epiphenomenon, as has been alluded to previously. ${ }^{32}$ Future studies evaluating the genetic and immune function profiles of such patients should help clarify these assumptions. Of patients treated with immunomodulatory therapy, $90 \%$ are currently alive with good control of their symptoms. Corticosteroids, either in the form of prednisone or budesonide, were the most commonly used immunomodulatory therapy. Steroids are accepted as a therapy for severe, poorly responsive celiac disease and refractory celiac disease. ${ }^{5}$ Budesonide is an attractive drug to treat patients with collagenous sprue, as it acts locally on the mucosa and has few systemic side effects. Used widely in Crohn's disease, it has been shown to benefit patients with poorly responsive celiac disease. ${ }^{42}$

The etiology of collagenous sprue is not known. Increases in procollagen I and tissue inhibitor of metalloproteinase-1 mRNAs, unopposed by increases in mRNA levels of matrix metalloproteinases 1 and 3 (key enzymes of extracellular matrix degradation), have been reported in collagenous sprue. ${ }^{32}$ Elevations of matrix metalloproteinases 1, 3 , and 9 and tissue inhibitor of metalloproteinase -1 proteins have been described in active celiac disease, indicating continual matrix remodeling in 
celiac disease. ${ }^{43}$ An imbalance between collagen synthesis and degradation might underlie increased subepithelial fibrosis, especially in celiac diseaseassociated collagenous sprue. No increase in smooth muscle antigen-positive myofibroblasts in our case corroborates a previous report that suggested increased synthesis and/or secretion of collagen, rather than an increase in cells producing it, in collagenous sprue patients. ${ }^{32}$ The increased number of eosinophils in biopsies displaying more severe grades of fibrosis suggests a role for these cells in the pathogenesis of collagenous sprue, as has been hypothesized in collagenous colitis and collagenous gastritis, ${ }^{44,45}$ as eosinophils are known to have profibrogenic effects. ${ }^{46}$ Contributions of, and interactions between, epithelial, inflammatory and stromal cell-derived inflammatory mediators, cytokines, and extracellular matrix components, implicated in inducing fibrosis in other inflammatory intestinal diseases, also need to be defined for collagenous sprue. ${ }^{47}$ Elucidating polymorphisms in genes leading to increased production of profibrogenic factors or increased sensitivity to them might also be worthwhile endeavors.

\section{Disclosure/conflict of interest}

The authors declare no conflict of interest.

\section{References}

1 Schein J. Syndrome of nontropical sprue with hitherto undescribed lesions of the intestine. Gastroenterology 1947;8:438-460.

2 Weinstein WM, Saunders DR, Tytgat GN, et al. Collagenous sprue-an unrecognized type of malabsorption. N Engl J Med 1970;283:1297-1301.

3 Barry RE, Morris JS, Read AE. Collagenous sprue. N Engl J Med 1971;284:1041.

4 Bossart R, Henry K, Booth CC, et al. Subepithelial collagen in intestinal malabsorption. Gut 1975;16:18-22.

5 Cellier C, Delabesse E, Helmer C, et al. Refractory sprue, coeliac disease, and enteropathy-associated T-cell lymphoma. French coeliac disease study group. Lancet 2000;356:203-208.

6 Doe WF, Evans D, Hobbs JR, et al. Coeliac disease, vasculitis, and cryoglobulinaemia. Gut 1972;13:112-123.

7 Freeman HJ. Collagenous sprue associated with an extensive T-cell lymphoma. J Clin Gastroenterol 2003;36:144-146.

8 Freeman HJ, Davis JE, Myers DM. Complete histological resolution of collagenous sprue. Can J Gastroenterol 2004;18:333-336.

9 McCashland TM, Donovan JP, Strobach RS, et al. Collagenous enterocolitis: a manifestation of glutensensitive enteropathy. J Clin Gastroenterol 1992;15: 45-51.

10 Pepper HW, Brandborg LL, Shanser JD, et al. Collagenous sprue. Am J Roentgenol Radium Ther Nucl Med 1974;121:275-282.
11 Robert ME, Ament ME, Weinstein WM. The histologic spectrum and clinical outcome of refractory and unclassified sprue. Am J Surg Pathol 2000;24:676-687.

12 Holdstock DJ, Oleesky S. Successful treatment of collagenous sprue with combination of prednisolone and gluten-free diet. Postgrad Med J 1973;49:664-667.

13 Sheehy TW, Staats OJ. Collagenous sprue. A case report. Ala J Med Sci 1972;9:392-396.

14 Woods CA, Foutch PG, Kerr DM, et al. Collagenous sprue as a cause for malabsorption in a patient with myotonic dystrophy: a new association. Am J Gastroenterol 1988;83:765-766.

15 Zeman RK, Toffler RB. The radiology corner: collagenous sprue. Am J Gastroenterol 1978;70:541-544.

16 Eckstein RP, Dowsett JF, Riley JW. Collagenous enterocolitis: a case of collagenous colitis with involvement of the small intestine. Am J Gastroenterol 1988;83:767-771.

17 Daniels JA, Lederman HM, Maitra A, et al. Gastrointestinal tract pathology in patients with common variable immunodeficiency (CVID): a clinicopathologic study and review. Am J Surg Pathol 2007;31: 1800-1812.

18 Freeman HJ, Berean KW. Resolution of paraneoplastic collagenous enterocolitis after resection of colon cancer. Can J Gastroenterol 2006;20:357-360.

19 Green PH. The many faces of celiac disease: clinical presentation of celiac disease in the adult population. Gastroenterology 2005;128:S74-S78.

20 Cellier C, Patey N, Mauvieux L, et al. Abnormal intestinal intraepithelial lymphocytes in refractory sprue. Gastroenterology 1998;114:471-481.

21 Daum S, Cellier C, Mulder CJ. Refractory coeliac disease. Best Pract Res Clin Gastroenterol 2005;19:413-424.

22 Ryan BM, Kelleher D. Refractory celiac disease. Gastroenterology 2000;119:243-251.

23 Oberhuber G, Granditsch G, Vogelsang H. The histopathology of coeliac disease: time for a standardized report scheme for pathologists. Eur J Gastroenterol Hepatol 1999;11:1185-1194.

24 Verbeek WH, Goerres MS, von Blomberg BM, et al. Flow cytometric determination of aberrant intraepithelial lymphocytes predicts T-cell lymphoma development more accurately than T-cell clonality analysis in refractory celiac disease. Clin Immunol 2008;126:48-56.

25 van Dongen JJ, Langerak AW, Bruggemann M, et al. Design and standardization of PCR primers and protocols for detection of clonal immunoglobulin and T-cell receptor gene recombinations in suspect lymphoproliferations: report of the BIOMED-2 Concerted Action BMH4-CT98-3936. Leukemia 2003;17:2257-2317.

26 Daum S, Weiss D, Hummel M, et al. Frequency of clonal intraepithelial $\mathrm{T}$ lymphocyte proliferations in enteropathy-type intestinal T cell lymphoma, coeliac disease, and refractory sprue. Gut 2001;49:804-812.

27 Lee SK, Green PH. Endoscopy in celiac disease. Curr Opin Gastroenterol 2005;21:589-594.

28 Cooke WT, Fone DJ, Cox EV, et al. Adult coeliac disease. Gut 1963;4:279-291.

29 Lazenby AJ, Yardley JH, Giardiello FM, et al. Pitfalls in the diagnosis of collagenous colitis: experience with 75 cases from a registry of collagenous colitis at the Johns Hopkins Hospital. Hum Pathol 1990;21:905-910.

30 Hopper AD, Cross SS, Sanders DS. Patchy villous atrophy in adult patients with suspected glutensensitive enteropathy: is a multiple duodenal biopsy strategy appropriate? Endoscopy 2008;40:219-224. 
31 Green PH. Celiac disease: how many biopsies for diagnosis? Gastrointest Endosc 2008;67:1088-1090.

32 Daum S, Foss HD, Schuppan D, et al. Synthesis of collagen I in collagenous sprue. Clin Gastroenterol Hepatol 2006;4:1232-1236.

33 Lagorce-Pages C, Fabiani B, Bouvier R, et al. Collagenous gastritis: a report of six cases. Am J Surg Pathol 2001;25:1174-1179.

34 Leung ST, Chandan VS, Murray JA, et al. Collagenous gastritis: histopathologic features and association with other gastrointestinal diseases. Am J Surg Pathol 2009;33:788-798.

35 Lee SK, Lo W, Memeo L, et al. Duodenal histology in patients with celiac disease after treatment with a gluten-free diet. Gastrointest Endosc 2003;57:187-191.

36 Akram S, Murray JA, Pardi DS, et al. Adult autoimmune enteropathy: Mayo Clinic Rochester experience. Clin Gastroenterol Hepatol 2007;5:1282-1290; quiz 1245.

37 Sategna Guidetti C, Solerio E, Scaglione N, et al. Duration of gluten exposure in adult coeliac disease does not correlate with the risk for autoimmune disorders. Gut 2001;49:502-505.

38 Ventura A, Magazzu G, Greco L. Duration of exposure to gluten and risk for autoimmune disorders in patients with celiac disease. SIGEP Study Group for Autoimmune Disorders in Celiac Disease. Gastroenterology 1999;117:297-303.
39 Pardi DS, Smyrk TC, Tremaine WJ, et al. Microscopic colitis: a review. Am J Gastroenterol 2002;97: 794-802.

40 Lo W, Sano K, Lebwohl B, et al. Changing presentation of adult celiac disease. Dig Dis Sci 2003;48:395-398.

41 Verkarre V, Asnafi V, Lecomte $\mathrm{T}$, et al. Refractory coeliac sprue is a diffuse gastrointestinal disease. Gut 2003;52:205-211.

42 Brar P, Lee S, Lewis S, et al. Budesonide in the treatment of refractory celiac disease. Am J Gastroenterol 2007;102:2265-2269.

43 Mohamed BM, Feighery C, Kelly J, et al. Increased protein expression of matrix metalloproteinases $-1,-3$, and -9 and TIMP-1 in patients with gluten-sensitive enteropathy. Dig Dis Sci 2006;51:1862-1868.

44 Levy AM, Yamazaki K, Van Keulen VP, et al. Increased eosinophil infiltration and degranulation in colonic tissue from patients with collagenous colitis. Am J Gastroenterol 2001;96:1522-1528.

45 Pulimood AB, Ramakrishna BS, Mathan MM. Collagenous gastritis and collagenous colitis: a report with sequential histological and ultrastructural findings. Gut 1999;44:881-885.

$46 \mathrm{Xu} \mathrm{X}$, Rivkind A, Pikarsky A, et al. Mast cells and eosinophils have a potential profibrogenic role in Crohn disease. Scand J Gastroenterol 2004;39:440-447.

47 Rieder F, Brenmoehl J, Leeb S, et al. Wound healing and fibrosis in intestinal disease. Gut 2007;56:130-139. 